International Journal of Information Technology \& Decision Making

Vol. 14, No. 6 (2015) 1401-1406

(C) World Scientific Publishing Company

DOI: $10.1142 / \mathrm{S} 0219622015990011$

\title{
Author Index Volume 14 (2015)
}

Akhand, M. A. H., see Shill, P. C.

14 (2015) 1063-1092

Alex, R., see Li, X.

Amiri, M., see Ghorabaee, M. K.

Asaduzzaman, M., see Shill, P. C.

Beigi, Z. G., see Hatami-Marbini, A.

14 (2015) 69-91

14 (2015) 993-1016

14 (2015) 1063-1092

14 (2015) 1189-1213

Borg, A., see Sariyar, M.

14 (2015) 521-533

Butenko, L. N., see Oleynikov, D. P.

14 (2015) 115-140

Cai, M., see $\mathrm{Yu}, \mathrm{X}$.

Cao, J., see $\mathrm{Yu}$, X.

Chang, C. C., see Chen, F. H.

Chang, C.-C., see Chen, P.-C.

Che, H.-L., see Wang, X.-Y.

Chen, C.-H., Hong, T.-P., Lee, Y.-C. and Tseng, V. S., Finding active membership functions for genetic-fuzzy data mining

Chen, F. H., Tzeng, G.-H. and Chang, C. C., Evaluating the enhancement of corporate social responsibility websites quality based on a new hybrid MADM model

Chen, N., Xu, Z. and Xia, M., The ELECTRE I multi-criteria decision-making method based on hesitant fuzzy sets

Chen, P.-C., Yu, M.-M., Chang, C.-C., Hsu, S.-H. and Managi, S., Nonradial directional performance measurement with undesirable outputs: An application to OECD and non-OECD countries

14 (2015) 915-942

14 (2015) 915-942

14 (2015) 697-724

14 (2015) 481-520

14 (2015) 901-913

14 (2015) 1215-1242

14 (2015) 697-724

14 (2015) 621-657

Chen, X., see Zhou, Y.

Cheng, J. T. S., Jiang, I.-M. and Liu, Y.-H., Technological innovation, product life cycle and market power: A real options approach

Cheng, W., see $\mathrm{Gu}, \mathrm{D}$.

Cho, S.-B., see Park, H.-S.

Choi, M.-J., see Lee, I.

Dai, W., see Tang, L.

Danielson, M., see Larsson, A.

De Brito, M. P., see Najafi, M.

Deng, S., see Zhou, L.

Di Caprio, D., Santos Arteaga, F. J. and Tavana, M., Choice manipulation through comparability in markets with verifiable multi-attribute products

Dullaert, W., see Najafi, M.

14 (2015) 339-374

14 (2015) 769-803

14 (2015) 93-113

14 (2015) 597-620

14 (2015) $317-338$

14 (2015) 395-420

14 (2015) $141-169$

14 (2015) 1299-1330

14 (2015) 769-803

14 (2015) 847-876

14 (2015) $769-803$ 
Ekenberg, L., see Larsson, A.

14 (2015) 1299-1330

Ergu, D., see Saaty, T. L.

Farahani, R. Z., see Najafi, M.

Francisco, L.-C., Francisco, M.-L. and Juan, S.-F., Payment systems in new electronic environments: Consumer behavior in payment systems via SMS

Francisco, M.-L., see Francisco, L.-C.

Fukushima, M., see Ong, B. T.

Fukuyama, H. and Weber, W. L., Network performance of Japanese credit cooperatives, 2004-2007

Fukuyama, H., see Hatami-Marbini, A.

Galarza-Molina, S. L., Torres, A., Moura, P. and Lara-Borrero, J., CRIDE: A case study in multi-criteria analysis for decisionmaking support in rainwater harvesting

Gelashvili, K., see Sirbiladze, G.

Ghobadi, S. and Jahangiri, S., Inverse DEA: Review, extension and application

Gholami, K., see Hatami-Marbini, A.

Ghorabaee, M. K., Amiri, M., Sadaghiani, J. S. and Zavadskas, E. K., Multi-criteria project selection using an extended VIKOR method with interval type-2 fuzzy sets

Granat, J., see Wierzbicki, A. P.

Gu, D., Liang, C., Kim, K.-S., Yang, C., Cheng, W. and Wang, J., Which is more reliable, expert experience or information itself? Weight scheme of complex cases for health management decision making

Gupta, M. M., see Song, K.-Y.

Hadavandi, E., see Tavakkoli, A.

Hakamipour, N. and Rezaei, S., Optimal design for a bivariate simple step-stress accelerated life testing model with type-II censoring and gompertz distribution

Halme, M. and Korhonen, P. J., Using value efficiency analysis to benchmark nonhomogeneous units

Hatami-Marbini, A., Beigi, Z. G., Fukuyama, H. and Gholami, K., Modeling centralized resources allocation and target setting in imprecise data envelopment analysis

Hong, T.-P., see Chen, C.-H.

Hsu, S.-H., see Chen, P.-C.

Huang, H.-Z., see Yang, J.-P.

Huang, J.-J. and Inuiguchi, M., Diminishing utility decision model for weighting criteria

Hunag, J., Yuan, X., Zhong, N. and Yao, Y., Modeling tag-aware recommendations based on user preferences

Inuiguchi, M., see Huang, J.-J.

Ivanov, V. I., Second- and first-order optimality conditions in vector optimization

Jahangiri, S., see Ghobadi, S.

Jiang, I.-M., see Cheng, J. T. S.

Juan, S.-F., see Francisco, L.-C.

Kandemir, C., see Özpeynirci, Ö.

14 (2015) 1171-1187

14 (2015) 769-803

14 (2015) 421-449

14 (2015) 421-449

14 (2015) 171-194

14 (2015) 825-846

14 (2015) 1189-1213

14 (2015) 43-67

14 (2015) 565-596

14 (2015) 805-824

14 (2015) 1189-1213

14 (2015) 993-1016

14 (2015) 1035-1062

14 (2015) 597-620

14 (2015) 659-696

14 (2015) 195-215

14 (2015) 1243-1262

14 (2015) 727-745

14 (2015) 1189-1213

14 (2015) 1215-1242

14 (2015) 481-520

14 (2015) 1017-1034

14 (2015) 1263-1284

14 (2015) 947-970

14 (2015) 1263-1284

14 (2015) 747-767

14 (2015) 805-824

14 (2015) 93-113

14 (2015) 421-449

14 (2015) 1111-1128 
Khutsishvili, I., see Sirbiladze, G.

14 (2015) 565-596

Kim, K.-S., see Gu, D.

14 (2015) 597-620

Korhonen, P. J., see Halme, M.

Kozinski, J. A., see Song, K.-Y.

Lara-Borrero, J., see Galarza-Molina, S. L.

Larsson, A., Riabacke, M., Danielson, M. and Ekenberg, L., Cardinal and rank ordering of criteria - addressing prescription within weight elicitation

14 (2015) $727-745$

14 (2015) 659-696

$14(2015) 43-67$

14 (2015) 1299-1330

14 (2015) 395-420

Lee, I., Lee, C.-K., Yoo, S. and Choi, M.-J., A decision-making model of social shopping in franchising: Assessing collaboration strategies

Lee, Y.-C., see Chen, C.-H.

Li, M., see Yang, H.

Li, X., Tian, Y., Smarandache, F. and Alex, R., An extension collaborative innovation model in the context of big data

Li, Y.-F., see Yang, J.-P.

Liagkouras, K. and Metaxiotis, K., Efficient portfolio construction with the use of multiobjective evolutionary algorithms: Best practices and performance metrics

Liang, C., see Gu, D.

Liang, L., see Zha, Y.

Liu, F., Shi, Y. and Wang, B., World search engine IQ test based on the internet IQ evaluation algorithms

Liu, X., see Zhou, L.

Liu, Y., see Yang, J.-P.

Liu, Y.-H., see Cheng, J. T. S.

Makowski, M., see Wierzbicki, A. P.

Malakooti, B., Double helix value functions, ordinal/cardinal approach, additive utility functions, multiple criteria, decision paradigm, process, and types ( $\mathrm{Z}$ theory I)

Managi, S., see Chen, P.-C.

Martens, B., see Walterbusch, M.

Mechitov, A. I., see Oleynikov, D. P.

Metaxiotis, K., see Liagkouras, K.

Mokhtari, H. and Salmasnia, A., An evolutionary clustering-based optimization to minimize total weighted completion time variance in a multiple machine manufacturing system

Mora, M., see Wang, F.

Moshkovich, H. M., see Oleynikov, D. P.

Moura, P., see Galarza-Molina, S. L.

Murase, K., see Shill, P. C.

Najafi, M., Farahani, R. Z., De Brito, M. P. and Dullaert, W., Location and distribution management of relief centers: $\mathrm{A}$ genetic algorithm approach

Najjar, L., see Wang, Y.

Oleynikov, D. P., Butenko, L. N., Moshkovich, H. M. and Mechitov, A. I., ARACE - A new method for verbal decision analysis

14 (2015) 395-420

14 (2015) 1215-1242

14 (2015) 287-315

$14(2015) 69-91$

14 (2015) 1017-1034

$14(2015) 535-564$

14 (2015) 597-620

14 (2015) 1285-1297

14 (2015) 221-237

14 (2015) 847-876

14 (2015) 1017-1034

14 (2015) 93-113

14 (2015) 1035-1062

14 (2015) 1353-1400

14 (2015) 481-520

14 (2015) 253-285

14 (2015) 115-140

14 (2015) 535-564

14 (2015) 971-991

14 (2015) 455-479

14 (2015) 115-140

14 (2015) 43-67

14 (2015) 1063-1092

14 (2015) 769-803

14 (2015) 239-251

14 (2015) 115-140 
Ong, B. T. and Fukushima, M., Automatically terminated particle swarm optimization with principal component analysis

Ong, H. C., see Tilahun, S. L.

Özdemir, M. S., see Saaty, T. L.

Özpeynirci, Ö. and Kandemir, C., A pseudo-polynomial time algorithm for a special multiobjective order picking problem

Park, H.-S., Park, M.-H. and Cho, S.-B., Mobile information recommendation using multi-criteria decision making with Bayesian network

Park, M.-H., see Park, H.-S.

Pathak, V. K., see Srivastava, V.

Raisinghani, M. S., see Wang, F.

Rezaeenour, J., see Tavakkoli, A.

Rezaei, S., see Hakamipour, N.

Riabacke, M., see Larsson, A.

Saaty, T. L. and Ergu, D., When is a decision-making method trustworthy? Criteria for evaluating multi-criteria decisionmaking methods

Saaty, T. L., Özdemir, M. S. and Shang, J. S., The rationality of punishment - measuring the severity of crimes: An AHP-based orders-of-magnitude approach

Sadaghiani, J. S., see Ghorabaee, M. K.

Salmasnia, A., see Mokhtari, H.

Santos Arteaga, F. J., see Di Caprio, D.

Sariyar, M. and Borg, A., Deterministic linkage as a preceding filter for other record linkage methods

Seniuk, G. T. G., see Song, K.-Y.

Shang, J. S., see Saaty, T. L.

Shen, D., see Wang, X.-Y.

Shi, Y., see Liu, F.

Shill, P. C., Akhand, M. A. H., Asaduzzaman, M. and Murase, K., Optimization of fuzzy logic controllers with rule base size reduction using genetic algorithms

Sikharulidze, A., see Sirbiladze, G.

Sirbiladze, G., Gelashvili, K., Khutsishvili, I. and Sikharulidze, A., Temporalized structure of bodies of evidence in the multi-criteria decision-making model

Smarandache, F., see Li, X.

Soleimani-Damaneh, M., Generalized convexity and characterization of (weak) pareto-optimality in nonsmooth multiobjective optimization problems

Song, K.-Y., Seniuk, G. T. G., Kozinski, J. A., Zhang, W.-J. and Gupta, M. M., An innovative fuzzy-neural decision analyzer for qualitative group decision making

Sornette, D., see Yukalov, V. I.

Srivastava, V., Tripathi, B. K. and Pathak, V. K., Hybrid computation model for intelligent system design by synergism of modified EFC with neural network

Sun, Y., see Yang, H.

Tang, J., see $\mathrm{Wu}, \mathrm{Z}$.

14 (2015) $171-194$

14 (2015) $1331-1352$

$14(2015) 5-16$

14 (2015) 1111-1128

14 (2015) $317-338$

14 (2015) 317-338

$14(2015)$ 17-41

14 (2015) 455-479

14 (2015) 195-215

14 (2015) 1243-1262

14 (2015) 1299-1330

14 (2015) 1171-1187

14 (2015) 5-16

14 (2015) 993-1016

14 (2015) 971-991

14 (2015) 339-374

14 (2015) 521-533

14 (2015) 659-696

14 (2015) 5-16

14 (2015) 901-913

14 (2015) 221-237

14 (2015) 1063-1092

14 (2015) 565-596

14 (2015) 565-596

14 (2015) 69-91

14 (2015) 877-899

14 (2015) 659-696

14 (2015) 1129-1166

14 (2015) 17-41

14 (2015) 287-315

14 (2015) 375-394 
Tang, L., Dai, W., Yu, L. and Wang, S., A novel CEEMD-based EELM ensemble learning paradigm for crude oil price forecasting

Tavakkoli, A., Rezaeenour, J. and Hadavandi, E., A novel forecasting model based on support vector regression and bat meta-heuristic (Bat-SVR): Case study in printed circuit board industry

Tavana, M., see Di Caprio, D.

Teuteberg, F., see Walterbusch, M.

Tian, Y., see Li, X.

Tilahun, S. L. and Ong, H. C., Prey-predator algorithm: A new metaheuristic algorithm for optimization problems

Torres, A., see Galarza-Molina, S. L.

Tripathi, B. K., see Srivastava, V.

Tseng, V. S., see Chen, C.-H.

Tzeng, G.-H., see Chen, F. H.

Walterbusch, M., Martens, B. and Teuteberg, F., A decision model for the evaluation and selection of cloud computing services: A first step towards a more sustainable perspective

Wang, B., see Liu, F.

Wang, F., Mora, M. and Raisinghani, M. S., Web-based decision support for e-business strategies: A balanced scorecard approach

Wang, J., see Gu, D.

Wang, S., see Tang, L.

Wang, X., see $\mathrm{Yu}, \mathrm{X}$.

Wang, X.-Y., Zhang, W., Xiong, X., Che, H.-L. and Shen, D., Information and bargaining power: Evidence from SME lending in China

Wang, Y. and Najjar, L., Factor neural network theory and its applications

Weber, W. L., see Fukuyama, H.

Wierzbicki, A. P., Makowski, M. and Granat, J., Robustness testing of model based multiple criteria decisions: Fundamentals and applications

$\mathrm{Wu}, \mathrm{Z}$. and Tang, J., Designing and reporting on computational experiments of multi-objective component selection algorithm

Xia, M., see Chen, N.

Xiong, X., see Wang, X.-Y.

$\mathrm{Xu}, \mathrm{X}$., see Zhou, Y.

$\mathrm{Xu}, \mathrm{X}$., see Zhou, L.

$\mathrm{Xu}, \mathrm{Z}$., see Chen, N.

Yang, C., see Gu, D.

Yang, H., Zheng, Q., Li, M. and Sun, Y., How to avoid herd behavior: A stochastic multi-choice scheduling algorithm and parameters analysis in grid scheduling

Yang, J.-P., Huang, H.-Z., Liu, Y. and Li, Y.-F., Quantification classification algorithm of multiple sources of evidence

Yao, Y., see Hunag, J.

Yoo, S., see Lee, I.
14 (2015) 141-169

$$
\begin{aligned}
& 14 \text { (2015) 195-215 } \\
& 14 \text { (2015) 339-374 } \\
& 14 \text { (2015) 253-285 } \\
& 14 \text { (2015) 69-91 }
\end{aligned}
$$

$14(2015) 253-285$

14 (2015) 221-237

14 (2015) 455-479

14 (2015) $597-620$

14 (2015) 141-169

14 (2015) 915-942

14 (2015) 901-913

14 (2015) 239-251

14 (2015) 825-846

14 (2015) 1035-1062

14 (2015) 375-394

14 (2015) 621-657

14 (2015) 901-913

14 (2015) 1093-1110

14 (2015) 847-876

14 (2015) 621-657

14 (2015) 597-620

14 (2015) 287-315

14 (2015) 1017-1034

14 (2015) 947-970

14 (2015) 395-420 
$\mathrm{Yu}, \mathrm{C}$., see Zhou, Y.

14 (2015) 1093-1110

$\mathrm{Yu}$, L., see Tang, L.

14 (2015) $141-169$

$\mathrm{Yu}, \mathrm{M} .-\mathrm{M}$. , see Chen, P.-C.

$\mathrm{Yu}$, X., Wang, X., Cao, J. and Cai, M., An ensemble differential

14 (2015) 481-520 evolution for numerical optimization

Yuan, X., see Hunag, J.

Yukalov, V. I. and Sornette, D., Role of information in decision making of social agents

14 (2015) 915-942

14 (2015) 947-970

$14(2015)$ 1129-1166

14 (2015) $993-1016$

14 (2015) 1285-1297

14 (2015) 901-913

14 (2015) 659-696

14 (2015) $287-315$

14 (2015) 947-970

Zhong, N., see Hunag, J.

Zhou, L., Xu, X., Deng, S. and Liu, X., Redesigning a supply chain distribution network: Formulation and genetic algorithm-based solution procedure

Zhou, Y., Chen, X., Xu, X. and Yu, C., A multi-product newsvendor problem with budget and loss constraints

$14(2015) 847-876$

14 (2015) 1093-1110 